\title{
Atividade reprodutiva de peixes (Teleostei) e o defeso da pesca de arrasto no litoral norte de Santa Catarina, Brasil ${ }^{1}$
}

\author{
Leda M. de Souza ${ }^{2,3} \&$ Paulo de T. Chaves ${ }^{2,4}$
}

${ }^{1}$ Contribuição número 1722 do Departamento de Zoologia, Universidade Federal do Paraná.
${ }^{2}$ Departamento de Zoologia, Universidade Federal do Paraná. Caixa Postal 19020, 81531-980 Curitiba, Paraná, Brasil.
${ }^{3}$ Bolsista da CAPES. E-mail: ledabio@yahoo.com.br
${ }^{4}$ Bolsista Produtividade em Pesquisa do CNPq. E-mail: ptchaves@ufpr.br

\begin{abstract}
Reproductive activity of fish (Teleostei) and closed season to shrimp trawling off ther northern coast of Santa Catarina, Brazil. Shrimp trawling was monitored in southern Brazil in order to study the reproductive status of teleosts occurring as by-catch. From 2005 to 2007 seventy-six fish species were found in this kind of fisheries. Gonad stages were evaluated and the Reproductive Activity Index was calculated. Mature individuals were $50 \%$ of the total caught as by-catch. Breeding activity in Isopisthus parvipinnis was "very high" in spring 2005 and summer 2007, and in a particular season (depending on the species) in Menticirrhus americanus, Stellifer sp., Pomadasys corvinaeformis, Stellifer brasiliensis, Syacium papillosum, Larimus breviceps, Diapterus rhombeus, Symphurus tessellatus, Chirocentrodon bleekerianus, Pellona harroweri, Anchoa tricolor, and Selene setapinnis. In the summer of 2007, followed by the spring of 2005 , trawling caught more species in "high" or "very high" breeding activity. In the summer of 2007 a larger number of species was in reproductive activity than the same season of 2006, due to a closed season in October-December 2006. Aiming to improve the fish breeding activity in this region, it is recommended to close this area for shrimp trawling during the spring. This measure could be positive for both species that spawn in spring and those preparing to spawn in the summer.
\end{abstract}

KEY WORDS. By-catch; closed season; small-scale fishery; spawning; teleosts.

RESUMO. Desembarques de arrasto visando à pesca do camarão sete-barbas, Xiphopenaeus kroyeri, foram acompanhados de 2005 a 2007 no município de São Francisco do Sul, Santa Catarina. Setenta e seis espécies de teleósteos foram registradas como captura incidental e seus indivíduos foram avaliados quanto ao estádio de maturação e Índice de Atividade Reprodutiva. Constatou-se que indivíduos de metade das espécies são capturados com gônadas maduras, em estações que variaram conforme a espécie. Em Isopisthus parvipinnis a atividade enquadrou-se na categoria "muito intensa" na primavera de 2005 e no verão de 2007; em Menticirrhus americanus, Stellifer sp., Pomadasys corvinaeformis, Stellifer brasiliensis, Syacium papillosum, Larimus breviceps, Diapterus rhombeus, Symphurus tessellatus, Chirocentrodon bleekerianus, Pellona harroweri, Anchoa tricolor e Selene setapinnis apenas em uma estação, dependendo da espécie. $O$ verão de 2007, seguido da primavera de 2005, foram as estações em que o arrasto incidiu sobre maior número de espécies em atividade reprodutiva "intensa" ou "muito intensa". No verão de 2007 o arrasto camaroeiro incidiu sobre um conjunto de espécies em atividade reprodutiva maior que na mesma estação em 2006. Atribui-se esse fato à proibição legal do arrasto de camarão no trimestre de outubro-dezembro de 2006, favorecendo a atividade reprodutiva das espécies na estação subseqüente. Recomenda-se que a gestão da pesca camaroeira na região mantenha o defeso de arrasto na primavera, assim beneficiando não apenas as espécies de peixes que desovam nesta estação, mas também aquelas que se preparam para a desova no verão.

PALAVRAS-CHAVE. Captura incidental; defeso; pesca artesanal; reprodução; teleósteos.

De longa data aponta-se o risco a que estão submetidas as populações de peixes costeiros como conseqüência de más práticas - aquelas que envolvem captura incidental - da pesca comercial (BerghaHn et al. 1992, VieIRA et al. 1996). O problema tem escala global (Diamond et al. 2000, HiLl \& WASSENBERg 2000), mas no sul do Brasil um motivo o faz alcançar uma dimensão particular: a freqüência de uso do arrasto camaroeiro em plataforma rasa, maior que noutras regiões do país (PAIVA 1997). De fato, pela natureza da rede e da forma como operam, as operações de arrasto têm um baixo grau de seletividade (Perez \& Pezzuto 1998, Diamond et al. 2000), fato que eleva a captura incidental.

Revista Brasileira de Zoologia 24 (4): 1113-1121, dezembro 2007 
No sul-sudeste do país a ocorrência de peixes como captura incidental da pesca de camarão está bem documentada (PAIVAFilho \& Schmiegelow 1986, Vieira et al. 1996, Kotas 1998, Chaves et al. 2003), entretanto o impacto associado ao ciclo de vida dessas espécies é ainda especulativo. Numa extensão litorânea de cerca de $60 \mathrm{~km}$ no norte de Santa Catarina e sul do Paraná (Fig. 1), dois programas de arrastos experimentais simulando a pesca de arrasto praticada em plataforma rasa já forneceram um inventário da ictiofauna exposta a tal tipo de pescaria: Branco \& VerAni (2006), entre Navegantes e Penha - 59 espécies de teleósteos e duas de raias; e Gomes \& CHAves (2006), entre Guaratuba e Itapoá - 60 espécies de teleósteos e uma de raia. Nenhum dos estudos avaliou a atividade reprodutiva da ictiofauna acompanhante, tampouco os efeitos associados à interrupção periódica legal (o chamado "defeso") da prática de arrasto. Anualmente um período de defeso é fixado na região, referenciado no ciclo de vida do camarão sete-barbas, Xiphopenaeus kroyeri Heller, 1862. Até 2005 o defeso ocorreu no outono, de abril a junho, mas em 2006 foi transferido para a primavera, de outubro a dezembro. O presente trabalho avalia a atividade reprodutiva dos peixes integrantes da captura incidental da pesca de arrasto de camarão e recomenda a melhor época para o defeso na região.

\section{MATERIAL E MÉTODOS}

Desembarques comerciais de arrasto camaroeiro foram acompanhados mensalmente na localidade de Enseada, município de São Francisco do Sul, no litoral norte de Santa Catarina (Fig. 1), de outubro de 2005 a março de 2007, com interrupção durante o defeso (outubro-dezembro de 2006). O arrasto na região tem como alvo o camarão sete-barbas. As redes, providas de pranchas ou portas, são tracionadas por canoas e botes de pequeno e médio portes, operando em profundidade de 4 a $20 \mathrm{~m}$ (Chaves \& Robert 2003). A primeira milha, profundidade de aproximadamente $2 \mathrm{~m}$, é interditada ao arrasto. Para grupamento dos dados segundo as estações do ano considerou-se primavera o trimestre outubro, novembro e dezembro, e assim sucessivamente, num total de cinco estações.

No presente estudo os peixes foram separados dos camarões e outros invertebrados e transportados sob refrigeração para laboratório, onde foram identificados, medidos (comprimento total - CT) e pesados (PT). O tamanho dos exemplares foi confrontado com o comprimento total máximo reconhecido para cada espécie, conforme descrito na literatura. Objetivou-se dessa forma obter um panorama do quão pequenos ou grandes são, relativamente à espécie em questão, os peixes da captura incidental.

O reconhecimento do sexo e estádio de maturação foi feito macroscopicamente (VAzzoler 1996): A) imaturo, B) em maturação, C) maduro, D) desovado ou espermiado. Consideraram-se "em reprodução" os indivíduos com gônadas em estádios C ou D. Os ovários foram a seguir retirados e pesados.

A reprodução foi avaliada com uso de três indicadores: (I) \% de indivíduos "em reprodução", ambos os sexos; (II) Índi-

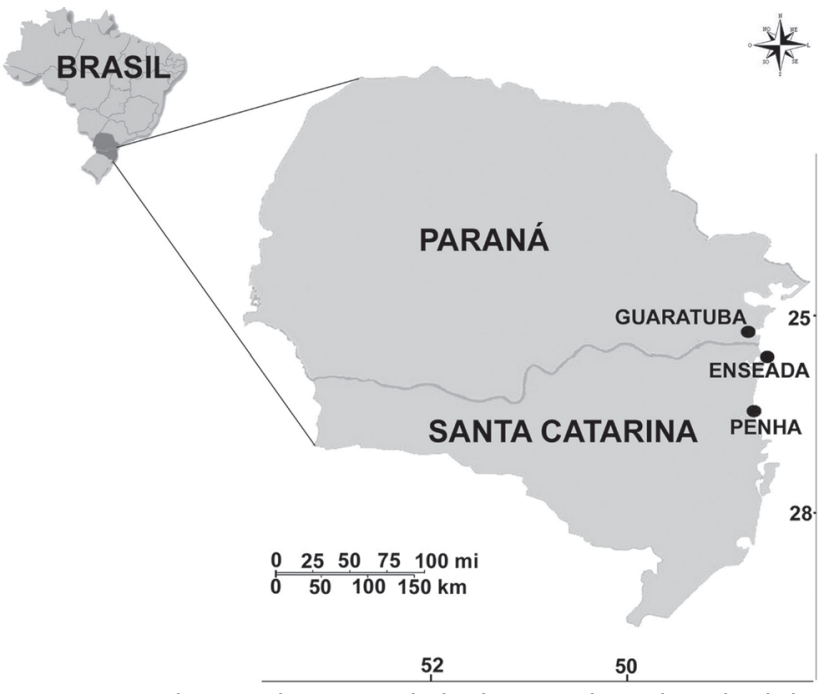

Figura 1. Localização da comunidade de Enseada no litoral sul do Brasil. Os pontos Guaratuba e Penha indicam comunidades junto às quais a captura incidental de arrasto foi estudada por GoMEs \& Chaves (2006) e Branco \& Verani (2006), respectivamente.

ce Gonadossomático das fêmeas: IGS = 100.PG/PT, sendo PG o peso dos ovários. Os valores de IGS foram utilizados no cálculo do Índice de Atividade Reprodutiva - IAR; e (III) Índice de Atividade Reprodutiva (IAR), desenvolvido por AgostinHo et al. (1993) apenas para fêmeas; espécies com n>30 em todo o período de estudo:

$$
\operatorname{IAR}=100 . \frac{\ln N_{i}\left(\left(n_{i} / \sum_{i: 1, n} n_{i}\right)+\left(n_{i} / N_{i}\right)\right) \cdot 1 I^{\prime} S_{i} / \text { IGS }_{e}}{\ln N_{m}\left(n_{m} / \sum_{i: 1, n} n_{i}+1\right)}
$$

sendo $\mathrm{N}_{\mathrm{i}} \mathrm{o}$ número de indivíduos na unidade amostral ' $\mathrm{i}$ ', $\mathrm{n}_{\mathrm{i}} \mathrm{O}$ número de indivíduos em reprodução na unidade amostral ' $i$ ', $\mathrm{N}_{\mathrm{m}}$ o número de indivíduos na maior unidade amostral, $\mathrm{n}_{\mathrm{m}} \mathrm{o}$ número de indivíduos em reprodução (estádios $\mathrm{C}$ ou $\mathrm{D}$ ) na maior unidade amostral, IGS o valor médio de IGS dos indivíduos em reprodução na unidade amostral 'i', e IGS o valor mais alto de IGS na unidade amostral ' $i$ '. Seguindo proposição de Agostinho et al. (1993), a atividade reprodutiva foi classificada em cinco categorias: "nula" quando IAR $\leqslant 2$, "incipiente" quando $2<\mathrm{IAR} \leqslant 5$, "moderada" quando $5<\mathrm{IAR} \leqslant 10$, "intensa" quando $10<\mathrm{IAR} \leqslant 20$, e "muito intensa" quando IAR $>20$.

\section{RESULTADOS}

Setenta e seis espécies de teleósteos foram identificadas acompanhando as capturas do arrasto camaroeiro (Tab. I). Além dessas, integrou as capturas um número impreciso de espécies do gênero Cynoscion, não identificadas no nível de espécie. Um terço das espécies esteve representado por indivíduos com ta- 
Tabela I. Espécies de teleósteos registradas na captura incidental da pesca de arrasto camaroeiro. (n) Número de exemplares analisados, (CT) seu comprimento total mínimo (Mín) e máximo (Máx), (Lit) comprimento máximo dos exemplares da espécie, segundo descrito na literatura, com sua fonte (F), (\%Máx) porcentagem do CT máximo analisado em relação ao CT Lit.

\begin{tabular}{|c|c|c|c|c|c|c|}
\hline \multirow{2}{*}{ Espécie } & \multirow{2}{*}{$\mathrm{n}$} & \multicolumn{3}{|c|}{$\mathrm{CT}(\mathrm{mm})$} & \multirow{2}{*}{ Fonte * } & \multirow{2}{*}{ \% Máx } \\
\hline & & Mín & Máx & Lit & & \\
\hline Achirus declivis Chabanaud, 1940 & 1 & 70 & 70 & 180 & 6 & 39 \\
\hline Anchoa lyolepis (Evermann \& Marsh, 1900) & 5 & 59 & 87 & 120 & 4 & 73 \\
\hline Anchoa spinifera Valenciennes, (1848) & 2 & 135 & 146 & 240 & 1 & 61 \\
\hline Anchoa tricolor Spix \& Agassiz, (1829) & 73 & 73 & 98 & 100 & 1 & 98 \\
\hline Anchovia clupeoides (Swainson, 1839) & 18 & 70 & 156 & 210 & 1 & 74 \\
\hline Anchoviella brevirostris (Günther, 1868) & 1 & 136 & 136 & 97 & 1 & 140 \\
\hline Astroscopus y-graecum (Cuvier, 1829) & 5 & 42 & 65 & 400 & 3 & 16 \\
\hline Bairdiella ronchus (Cuvier, 1830) & 212 & 49 & 150 & 350 & 2 & 43 \\
\hline Cathorops spixii (Agassiz, 1829) & 2 & 139 & 139 & 300 & 6 & 46 \\
\hline Centropomus parallelus Poey, 1860 & 4 & 211 & 240 & 600 & 2 & 40 \\
\hline Centropomus undecimalis Bloch, 1792 & 2 & 229 & 267 & 400 & 2 & 67 \\
\hline Chaetodipterus faber (Broussonet, 1782) & 3 & 33 & 105 & 900 & 3 & 12 \\
\hline Chilomycterus spinosus spinosus (Linnaeus, 1758) & 3 & 28 & 70 & 250 & 6 & 28 \\
\hline Chirocentrodon bleekerianus (Poey, 1867) & 495 & 33 & 141 & 111 & 1 & 127 \\
\hline Chloroscombrus chrysurus (Linnaeus, 1766) & 53 & 36 & 150 & 300 & 2 & 50 \\
\hline Citharichthys arenaceus Evermann \& Marsh, 1900 & 25 & 110 & 186 & 150 & 5 & 124 \\
\hline Citharichthys spilopterus Gunther, 1862 & 6 & 130 & 164 & 180 & 5 & 91 \\
\hline Conodon nobilis (Linnaeus, 1758) & 30 & 66 & 193 & 320 & 2 & 60 \\
\hline Ctenosciaena gracilicirrhus (Metzelaar, 1919) & 1 & 148 & 148 & 210 & 2 & 70 \\
\hline Dactylopterus volitans (Linnaeus, 1758) & 5 & 89 & 230 & 450 & 2 & 51 \\
\hline Diapterus auratus Ranzani, 1842 & 5 & 106 & 171 & 350 & 2 & 49 \\
\hline Diapterus rhombeus (Cuvier, 1829) & 56 & 105 & 197 & 400 & 2 & 49 \\
\hline Diplectrum radiale (Quoy \& Gaimard, 1824) & 1 & 184 & 184 & 260 & 4 & 71 \\
\hline Etropus crossotus Jordan \& Gilbert, 1822 & 23 & 55 & 155 & 170 & 5 & 91 \\
\hline Eucinostomus gula (Cuvier, 1830) & 9 & 149 & 180 & 250 & 2 & 72 \\
\hline Eucinostomus melanopterus (Bleeker, 1863) & 3 & 139 & 234 & 227 & 2 & 103 \\
\hline Genidens barbus (Lacepède, 1803) & 2 & 175 & 250 & 1000 & 1 & 25 \\
\hline Gobiesox barbatulus Starks, 1913 & 1 & 53 & 53 & 85 & 1 & 62 \\
\hline Gymnothorax ocellatus Agassiz, 1834 & 2 & 370 & 380 & 900 & 6 & 42 \\
\hline Harengula clupeola (Cuvier, 1829) & 3 & 160 & 166 & 180 & 6 & 92 \\
\hline Hemicaranx amblyrhynchus (Cuvier, 1833) & 3 & 70 & 90 & 450 & 4 & 20 \\
\hline Isopisthus parvipinnis (Cuvier, 1830) & 486 & 45 & 194 & 250 & 2 & 78 \\
\hline Lagocephalus laevigatus (Linnaeus, 1766) & 25 & 56 & 110 & 600 & 5 & 18 \\
\hline Larimus breviceps Cuvier, 1830 & 829 & 32 & 200 & 300 & 2 & 67 \\
\hline Licengraulis grossidens (Agassiz, 1829) & 1 & 122 & 122 & 270 & 1 & 45 \\
\hline Macrodon ancylodon (Block \& Schneider, 1801) & 18 & 60 & 175 & 450 & 2 & 39 \\
\hline Menticirrhus americanus (Linné, 1758) & 144 & 70 & 261 & 500 & 2 & 52 \\
\hline Menticirrhus littoralis (Holbrook, 1860) & 8 & 90 & 166 & 450 & 2 & 37 \\
\hline Micropogonias furnieri (Desmarest, 1823) & 47 & 70 & 274 & 600 & 2 & 46 \\
\hline Myrophis punctatus Lütken, 1852 & 2 & 495 & 510 & 353 & 6 & 144 \\
\hline Ophichthus gomesii (Castelnau, 1855) & 8 & 477 & 595 & 760 & 4 & 78 \\
\hline Ophichthus parilis (Richardson, 1848) & 1 & 471 & 471 & 1000 & 1 & 47 \\
\hline
\end{tabular}


Tabela I. Continuação.

\begin{tabular}{|c|c|c|c|c|c|c|}
\hline \multirow{2}{*}{ Espécie } & \multirow{2}{*}{$\mathrm{n}$} & \multicolumn{3}{|c|}{$\mathrm{CT}(\mathrm{mm})$} & \multirow{2}{*}{ Fonte * } & \multirow{2}{*}{ \% Máx } \\
\hline & & Mín & Máx & Lit & & \\
\hline Ophidion holbrooki (Putnam, 1874) & 1 & 225 & 225 & 270 & 1 & 83 \\
\hline Ophioscion punctatissimus Meek \& Hildebrand, 1925 & 1 & 155 & 155 & 250 & 2 & 62 \\
\hline Opisthonema oglinum (Lesueur, 1817) & 1 & 185 & 185 & 380 & 4 & 49 \\
\hline Orthopristis ruber (Cuvier, 1830) & 7 & 168 & 265 & 400 & 2 & 66 \\
\hline Paralonchurus brasiliensis (Steindachner, 1875) & 492 & 41 & 250 & 300 & 2 & 83 \\
\hline Pellona harroweri (Fowler, 1917) & 361 & 36 & 246 & 160 & 1 & 154 \\
\hline Peprilus paru (Linnaeus, 1758) & 5 & 32 & 101 & 300 & 5 & 34 \\
\hline Platanichthys platana (Regan, 1917) & 1 & 124 & 124 & 90 & 1 & 138 \\
\hline Polydactylus virginicus (Linnaeus, 1758) & 9 & 103 & 190 & 320 & 3 & 59 \\
\hline Pomadasys corvinaeformis (Steindachner, 1868) & 70 & 66 & 214 & 250 & 2 & 86 \\
\hline Porichthys porosissimus (Cuvier, 1829) & 19 & 53 & 265 & 320 & 6 & 83 \\
\hline Prionotus punctatus (Bloch, 1797) & 18 & 50 & 272 & 500 & 2 & 54 \\
\hline Rypticus randalli Courtenay, 1967 & 22 & 109 & 176 & 200 & 6 & 88 \\
\hline Sardinella janeiro (Eigenmann, 1894) & 1 & 149 & 149 & 250 & 4 & 60 \\
\hline Selene setapinnis (Mitchill, 1815) & 144 & 31 & 195 & 400 & 2 & 49 \\
\hline Selene vomer (Linnaeus, 1758) & 15 & 31 & 90 & 500 & 2 & 18 \\
\hline Sphoeroides greeleyi (Gilbert, 1900) & 2 & 36 & 46 & 170 & 5 & 27 \\
\hline Sphoeroides testudineus (Linnaeus, 1758) & 11 & 50 & 85 & 250 & 5 & 34 \\
\hline Sphyraena guachancho Cuvier, 1829 & 2 & 308 & 331 & 1000 & 3 & 33 \\
\hline Stellifer brasiliensis (Schultz, 1945) & 768 & 45 & 190 & 180 & 2 & 106 \\
\hline Stellifer rastrifer (Jordan, 1889) & 807 & 39 & 200 & 200 & 2 & 100 \\
\hline Stellifer sp. ** & 256 & 45 & 165 & 100 & 2 & 165 \\
\hline Stellifer stellifer (Bloch, 1790) & 8 & 67 & 101 & 150 & 2 & 67 \\
\hline Stephanolepis hispidus (Linnaeus, 1766) & 1 & 202 & 202 & 275 & 6 & 73 \\
\hline Syacium micrurum Ranzani, 1842 & 3 & 86 & 175 & 236 & 5 & 74 \\
\hline Syacium papillosum (Linnaeus, 1758) & 40 & 80 & 239 & 260 & 5 & 92 \\
\hline Symphurus jenynsi (Evermann, 1906) & 1 & 158 & 158 & 319 & 6 & 50 \\
\hline Symphurus tessellatus (Quoy \& Gaimard, 1824) & 64 & 82 & 200 & 230 & 4 & 87 \\
\hline Synodus foetens (Linnaeus, 1766) & 2 & 185 & 224 & 430 & 4 & 52 \\
\hline Trichiurus lepturus Linnaeus, 1758 & 141 & 70 & 940 & 1400 & 5 & 67 \\
\hline Trinectes microphthalmus (Chabanaud, 1928) & 5 & 75 & 85 & 85 & 6 & 100 \\
\hline Trinectes paulistanus (Ribeiro, 1915) & 4 & 75 & 144 & 180 & 6 & 80 \\
\hline Umbrina coroides Cuvier, 1830 & 4 & 111 & 200 & 350 & 2 & 57 \\
\hline Urophycis brasiliensis (Kaup, 1858) & 28 & 35 & 200 & 600 & 1 & 33 \\
\hline
\end{tabular}

*Fonte: 1) Figueiredo \& Menezes (1978), 2) Figueiredo \& Menezes (1980), 3) Menezes \& Figueiredo (1980), 4) Cervigón et al. (1992), 5) Figueiredo \& Menezes (2000), 6) Fishbase. ** Stellifer sp. é apresentada em Menezes \& Figueiredo (1980), e novamente referida em Menezes et al. (2003), como espécie ainda não descrita.

manho inferior a 50\% do máximo registrado na literatura para elas, sendo em oito com tamanho inferior a 30\% do máximo. Por outro lado, em nove espécies foram registrados indivíduos excepcionalmente grandes, com tamanho superior ao máximo descrito na literatura (Tab. I).

Metade das espécies sobre as quais a pesca camaroeira incidiu encontrava-se em reprodução. Num primeiro grupo, constituído por 57 espécies que no total das amostras estiveram representadas por no máximo 30 exemplares, indivíduos com gônadas maduras foram detectados em vinte e duas espécies (Tab. II). Tais indivíduos tinham porte relativamente grande, superior - exceto em Sphoeroides testudineus e Sphyraena guachancho - a 50\% do máximo registrado na literatura para a espécie (Tab. I). Nesse grupo a pesca atingiu ainda outras 10 
Tabela II. Distribuição porcentual dos estádios de maturação gonadal dos teleósteos analisados com $\mathrm{n} \leqslant 30$ na captura incidental do arrasto camaroeiro, segundo o sexo e a espécie. Indeterminado: exemplares com sexo e estádio de maturação não-reconhecidos. Tamanho das amostras conforme tabela I.

\begin{tabular}{|c|c|c|c|}
\hline Espécie & Nome comum & Machos & Fêmeas \\
\hline Achirus declivis & Linguado & Indeterminado & \\
\hline Anchoa lyolepis & Manjuba & - & $60 \% \mathrm{~B} ; 40 \% \mathrm{C}$ \\
\hline Anchoa spinifera & Manjuba & Indeterminado & \\
\hline Anchovia clupeoides & Manjuba & $6 \% \mathrm{~B}$ & $94 \% \mathrm{~A}$ \\
\hline Anchoviella brevirostris & Manjuba & $100 \% \mathrm{~B}$ & - \\
\hline Astroscopus y-graecum & Miracéu & Indeterminado & \\
\hline Cathorops spixii & Bagre-amarelo & - & \\
\hline Centropomus parallelus & Robalo-peva & $100 \% \mathrm{~B}$ & - \\
\hline Centropomus undecimalis & Robalo-flexa & $100 \% \mathrm{~B}$ & - \\
\hline Chaetodipterus faber & Paru, enxada & Indeterminado & \\
\hline Chilomycterus spinosus & Baiacu-de-espinho & Indeterminado & \\
\hline Citharichthys arenaceus & Linguado & - & $72 \% \mathrm{~A} ; 12 \% \mathrm{~B} ; 16 \% \mathrm{C}$ \\
\hline Citharichthys spilopterus & Linguado & - & $17 \% A ; 33 \% B ; 50 C$ \\
\hline Conodon nobilis & Roncador & $25 \% A ; 25 \% C$ & $50 \% A$ \\
\hline Ctenosciaena gracilicirrhus & Cangauá & $100 \% \mathrm{C}$ & - \\
\hline Dactylopterus volitans & Voador, coió & - & $100 \% \mathrm{~B}$ \\
\hline Diapterus auratus & Carapeba & $60 \% A$ & $40 \% \mathrm{C}$ \\
\hline Diplectrum radiale & Michole & - & $100 \% \mathrm{C}$ \\
\hline Etropus crossotus & Linguado & - & $87 \% \mathrm{~A} ; 13 \% \mathrm{C}$ \\
\hline Eucinostomus gula & Escrivão, carapicu & $11 \% \mathrm{~B} ; 11 \% \mathrm{C}$ & $78 \% \mathrm{C}$ \\
\hline Eucinostomus & Escrivão & $34 \% \mathrm{~B}$ & $33 \% A ; 33 \% \mathrm{C}$ \\
\hline Genidens barbus & Bagre-branco & $100 \% B$ & - \\
\hline Gobiesox barbatulus & Pregador & Indeterminado & \\
\hline Gymnothorax ocellatus & Moréia-pintada & - & $100 \% A$ \\
\hline Harengula clupeola & Sardinha-cascuda & - & $100 \% \mathrm{C}$ \\
\hline Hemicaranx amblyrhynchus & Fede-fede & Indeterminado & \\
\hline Lagocephalus laevigatus & Baiacu & - & $100 \% A$ \\
\hline Licengraulis grossidens & Manjubão & - & $100 \% \mathrm{C}$ \\
\hline Macrodon ancylodon & Pescada-foguete & Indeterminado & \\
\hline Menticirrhus littoralis & Betara & $50 \% A$ & $50 \% A$ \\
\hline Myrophis punctatus & Pixe-cobra & $100 \% \mathrm{~B}$ & - \\
\hline Ophichthus gomesii & Peixe-cobra & - & $12 \% \mathrm{~A} ; 12 \% \mathrm{~B} ; 76 \% \mathrm{C}$ \\
\hline Ophichthus parilis & Peixe-cobra & Indeterminado & \\
\hline Ophidion holbrooki & Peixe-cobra & Indeterminado & \\
\hline Ophioscion punctatissimus & Canguá & - & $100 \% A$ \\
\hline Opisthonema oglinum & Sardinha-bandeira & - & $100 \% \mathrm{~B}$ \\
\hline Orthopristis ruber & Corcoroca & 13\%B;29\%C & $29 \% A ; 29 \% C$ \\
\hline Peprilus paru & Gordinho & Indeterminado & \\
\hline Platanichthys platana & Sardinha & - & $100 \% \mathrm{~A}$ \\
\hline Polydactylus virginicus & Parati-barbudo & Indeterminado & \\
\hline Porichthys porosissimus & Mamangá liso & $53 \% A$ & $10 \% A ; 37 \% \mathrm{C}$ \\
\hline Prionotus punctatus & Cabrinha & - & $100 \% C$ \\
\hline Rypticus randalli & Peixe-sabão & $5 \% \mathrm{C}$ & $45 \% \mathrm{~A} ; 18 \% \mathrm{~B} ; 32 \% \mathrm{C}$ \\
\hline
\end{tabular}


Tabela II. Continuação.

\begin{tabular}{llll}
\hline & \multicolumn{1}{c}{ Espécie } & \multicolumn{1}{c}{ Machos } & Fêmeas \\
\hline Sardinella janeiro & Sardinha & Indeterminado & \\
Selene vomer & Peixe-galo-de-penacho & Indeterminado & \\
Sphoeroides greeleyi & Baiacu & Indeterminado & $91 \% \mathrm{~A} ; 9 \% \mathrm{C}$ \\
Sphoeroides testudineus & Baiacu & - & - \\
Sphyraena guachancho & Bicuda & $50 \% \mathrm{~B} ; 50 \% \mathrm{C}$ & $67 \% \mathrm{~A} ; 33 \% \mathrm{~B}$ \\
Stellifer stellifer & Cangulo & - & - \\
Stephanolepis hispidus & Peixe-porco & Indeterminado & $100 \% \mathrm{~B}$ \\
Syacium micrurum & Linguado & $100 \% \mathrm{C}$ & $100 \% \mathrm{C}$ \\
Symphurus jenynsi & Linguado & Indeterminado & $100 \% \mathrm{C}$ \\
Synodus foetens & Peixe-lagarto & - & $100 \% \mathrm{~A}$ \\
Trinectes microphthalmus & Linguado & - & \\
Trinectes paulistanus & Linguado & - & \\
Umbrina coroides & Castanha & Indeterminado & \\
Urophycis brasiliensis & Abrótea & & \\
\hline
\end{tabular}

espécies em maturação gonadal (Tab. II). Nas demais, ou os indivíduos estavam apenas no estádio A, ou seu sexo e estádio de maturação não foram reconhecidos, sinalizando condição jovem. Num segundo grupo, constituído por 19 espécies que no total das amostras estiveram representadas por mais de 30 exemplares, indivíduos com gônadas maduras foram detectados em 16 delas (Tab. III).

Atividade reprodutiva (IAR $>2$ ) foi registrada ao longo de todo o período de estudo em estações que variaram conforme a espécie (Fig. 2). Somente numa das espécies - Isopisthus parvipinnis - a atividade enquadrou-se na categoria "muito intensa" em duas estações - a primavera de 2005 e o verão de 2007. Em outras 12 - Menticirrhus americanus, Stellifer sp., Pomadasys corvinaeformis, Stellifer brasiliensis, Syacium papillosum, Larimus breviceps, Diapterus rhombeus, Symphurus tessellatus, Chirocentrodon bleekerianus, Pellona harroweri, Anchoa tricolor e Selene setapinnis - a atividade reprodutiva foi "muito intensa" em apenas uma estação (Fig. 2). O arrasto incidiu no máximo na categoria "intensa" em três espécies: Stellifer rastrifer, Paralonchurus brasiliensis e Trichiurus lepturus (Fig. 2). Finalmente, os valores de IAR indicaram atividade reprodutiva "nula" em Bairdiella ronchus e Micropogonias furnieri em todas as estações em que ocorreram (Fig. 2). Parte das espécies identificadas como apresentando atividade reprodutiva "intensa" ou "muito intensa" tinha porcentagem de exemplares em reprodução inferior a $10 \%$, porém incluía exemplares de porte relativamente grande (Tab. II).

O verão de 2007 foi a estação em que o arrasto incidiu sobre maior número (11) de espécies em atividade reprodutiva "intensa" ou "muito intensa". Foi a época que sucedeu imediatamente o defeso de 2006, pela primeira vez realizado na primavera (Fig. 3). Seguiram-se em número de espécies em atividade reprodutiva a primavera de 2005 (6) e o verão e outono de 2006 (duas cada), nenhuma dessas estações sucedendo perí-

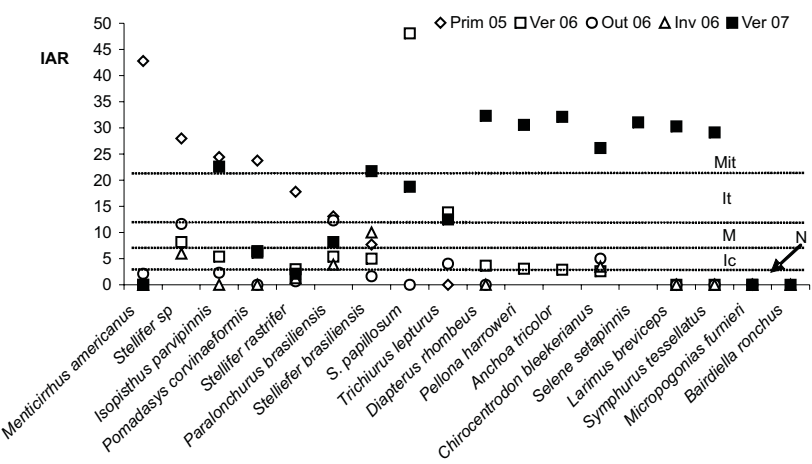

Figura 2. Distribuição de valores do Índice de Atividade Reprodutiva (IAR) por espécie integrante da captura incidental do arrasto camaroeiro, segundo a estação - primavera de 2005 a verão de 2007. (N) Nula, (IC) incipiente, (M) moderada, (I) intensa, (Mit) muito intensa.

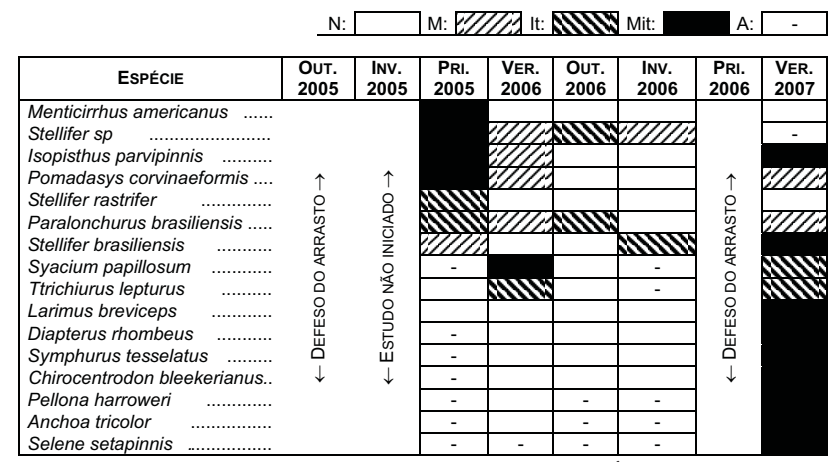

Figura 3. Distribuição sazonal das categorias de Índice de Atividade Reprodutiva nas espécies com Índice diferente de zero, outono de 2005 a verão de 2007. (N) Nula ou incipiente, (M) moderada, (It) intensa, (Mit) muito intensa, (A) espécie ausente na estação. 
Tabela III. Abundância porcentual (\%E.R.) de teleósteos em reprodução, estádios C + D, na captura incidental do arrasto camaroeiro, segundo a espécie ( $n>30$, conforme tabela I). Atividade reprodutiva: maior categoria de atividade reprodutiva conforme valores do Índice de Atividade Reprodutiva (IAR).

\begin{tabular}{llcc}
\hline \multicolumn{1}{c}{ Espécie } & Nome comum & \%E.R. & Atividade reprodutiva \\
\hline Anchoa tricolor & Manjuba & 44 & Muito intensa \\
Bairdiella ronchus & Roncador, oveva & 0 & Nula \\
Chirocentrodon bleekerianus & Sardinha-charuto & 38 & Muito intensa \\
Chloroscombrus chrysurus & Palombeta & 0 & * \\
Diapterus rhombeus & Carapeba & 21 & Muito intensa \\
Isopisthus parvipinnis & Pescadinha & 13 & Muito intensa \\
Larimus breviceps & Oveva & 2 & Muito intensa \\
Menticirrhus americanus & Betara & 3 & Muito intensa \\
Micropogonias furnieri & Corvina & 0 & Nula \\
Paralonchurus brasiliensis & Maria-luísa & 12 & Intensa \\
Pellona harroweri & Sardinha & 32 & Muito intensa \\
Pomadasys corvinaeformis & Corcoroca & 40 & Muito intensa \\
Selene setapinnis & Peixe-galo & $<1$ & Muito intensa \\
Stellifer brasiliensis & Cangulo & 14 & Muito intensa \\
Stellifer rastrifer & Cangulo & 49 & Intensa \\
Stellifer sp. & Cangulo & 5 & Muito intensa \\
Syacium papillosum & Linguado & 8 & Muito intensa \\
Symphurus tessellatus & Linguado & 61 & Muito intensa \\
Trichiurus lepturus & Espada & & Intensa \\
\hline nao identificado; especie & &
\end{tabular}

* Sexo não identificado; espécie não utilizada para cálculo de IAR.

odo de defeso (Fig. 3). No outono e no inverno de 2006 o arrasto não incidiu sobre nenhuma espécie com atividade "muito intensa", e sobre apenas duas ou uma na categoria "intensa", respectivamente (Fig. 3). De fato, no outono de 2006 o valor médio de IAR foi 2,8 (desvio-padrão, $\mathrm{S}=4,2$ ) e no inverno de 2005 foi 1,8 $(S=3,1)$, enquanto na primavera de 2005 foi 14,3 ( $\mathrm{S}=14,4)$, no verão de 2006 6,7 ( $=11,6)$ e no verão de 2007 foi $17,9(\mathrm{~S}=12,8)$.

Salientou-se também uma diferença entre os verões de 2006 e 2007. Considerando-se 12 espécies de ocorrência comum a ambas as estações e com valores de IAR diferentes de zero, a categoria de atividade reprodutiva foi semelhante entre os verões em três espécies - P. corvinaeformis, $P$. brasiliensis e $T$. lepturus, e superior no verão em oito espécies (Fig. 3). Assim, no verão de 2007 metade das espécies analisadas quanto ao IAR encontrava-se em atividade reprodutiva na categoria "muito intensa", situação similar à da primavera de 2005 mas diferente daquela do verão de 2006, quando apenas 12,5\% das espécies enquadravam-se em tal categoria.

\section{DISCUSSÃO}

O grau de risco que a pesca de arrasto impõe à conservação das populações de peixes já foi reportado em numerosos trabalhos. Estudos de WALter \& BeCKer (1994) e DiAmond et al. (1999), por exemplo, quantificaram em mais que 60\% a biomassa de peixes na captura incidental, trazendo conseqüências que não alcançam apenas o plano ambiental, mas também o econômico: segundo BERGHAHN et al. (1992), estoques de gadiformes no Mar do Norte foram comprometidos pela captura de juvenis como fauna acompanhante de pescarias de arrasto, o mesmo mais tarde estimado por Diamond et al. (2000) em cianídeos do Atlântico Norte.

Tais fatos remetem à questão: a captura incidental é mais danosa ao retirar do meio indivíduos jovens, ou ao retirar indivíduos que já adultos, mas estão em reprodução? Seja qual for a resposta, no presente trabalho ambos as situações foram identificadas: metade das espécies de peixes registradas na captura incidental não apresentou indícios de atividade reprodutiva, e metade, sim. Dentre as possíveis razões para o primeiro caso estão: (I) a época em que os indivíduos ocorrem na região, nãocoincidente com aquela de desova; (II) o fato destes ainda serem jovens, pré-maturação, hipótese favorecida pelo tamanho relativamente pequeno com que - ao menos em parte das espécies eles ocorrem nos arrastos; e/ou (III) a quantidade relativamente pequena de ocorrência nas amostras. Por outro lado, mesmo em algumas espécies com pequeno tamanho de amostra (ex.: $D$. radiale e L. grossidens) exemplares em reprodução foram encontrados. Tratam-se, possivelmente, de espécies pouco abundantes na região, ou de baixa vulnerabilidade à rede de fundo, mas que nem por isso estão isentas de terem a desova afetada pelo arrasto 
camaroeiro. Em outras sete espécies - L. breviceps, M. americanus, S. setapinnis, Stellifer sp., S. papillosum, S. tessellatus e T. lepturus a porcentagem de exemplares em reprodução foi muito baixa, inferior a 10\%; mesmo assim, apresentaram evidências de estar em atividade reprodutiva "intensa" ou "muito intensa".

Algumas espécies em que a atividade reprodutiva foi diagnosticada como sendo 'muito intensa' estiveram representadas em quantidade pequena, relativamente a outras, da mesma categoria. Numa primeira leitura tem-se que o arrasto estaria afetando menos as espécies do primeiro grupo que as do segundo, mais abundantes. Por outro lado, deve-se considerar que para manter-se no ambiente as espécies dependem de um tamanho mínimo de estoque. Uma vez que tal variável depende de atributos biológicos diferentes entre as espécies, resulta que, comparadas duas de mesma categoria reprodutiva, o impacto causado pelo arrasto pode estar sendo até maior sobre aquelas que são menos comuns no arrasto, supostamente menos abundantes na região, que sobre as mais comuns.

Nos trabalhos que relatam a composição ictiofaunística de arrastos camaroeiros no litoral sul do Paraná e norte de Santa Catarina (Chaves et al. 2003, Branco \& Verani 2006, Gomes \& Chaves 2006) é comum a menção a raias, aqui não encontradas. Por outro lado, o número de espécies componentes da fauna acompanhante foi superior no presente acompanhamento (76) àqueles dos três trabalhos citados (61 ou 62). Esse fato, provável decorrência de diferenças de esforço amostral e de profundidades de operação, sinaliza que - numa mesma região - o impacto do arrasto camaroeiro sobre a reprodução da ictiofauna pode variar segundo a distância das operações em relação à costa.

Verão e primavera como época reprodutiva de peixes é fato conhecido em estuários e plataforma do litoral norte de Santa Catarina e sul do Paraná, incluindo-se cinco espécies aqui registradas na captural incidental do arrasto: $C$. arenaceus e $C$. spilopterus (Chaves \& Vendel 1997a), S. rastrifer (Chaves \& Vendel 1997b), I. parvipinnis (CHAves et al. 1998) e C. bleekerianus (CorrêA et al. 2005). Nesta região há grandes extensões de estuários, relativamente bem preservados, e tradicionalmente aceita-se que as áreas estuarinas atuem como berçário para espécies de plataforma (BLABER 2000). Entretanto, a maior parte das espécies que utilizam os estuários da região para desova ou como berçário (relações disponíveis em Chaves \& Bouchereau 2000 e Vendel \& Chaves 2006) não coincide com aquelas que no presente trabalho foram registradas em atividade reprodutiva. Duas dentre as espécies que se reproduzem na região - G. barbus (CHAves \& Bouchereau 2000) e M. furnieri (Robert \& Chaves 2001) não são capturadas no arrasto camaroeiro em tal situação. Por outro lado, nos estuários não é conhecida desova de $P$. corvinaeformis, e CHAVEs \& CORRÊA (2000) haviam proposto que os indivíduos utilizam o manguezal da Baía de Guaratuba em fase anterior à de maturação para depois desovar na plataforma. O presente trabalho, ao constatar atividade reprodutiva muito intensa em $P$. corvinaeformis no arrasto de plataforma, confirma essa hipótese. Assim, pode-se estimar que a própria área de plataforma rasa onde ocorre o arrasto camaroeiro seja local de desova e de criação de certo número de espécies que não utilizam os estuários para tal, fato que sublinha o papel desse ambiente na conservação de recursos pesqueiros.

No verão de 2007 o arrasto camaroeiro incidiu sobre uma ictiofauna em atividade reprodutiva mais expressiva (em número de espécies e em valores de IAR) que aquela do verão de 2006. A razão disso provavelmente tenha sido a ausência de arrastos durante o defeso no trimestre outubro-dezembro de 2006, favorecendo a atividade reprodutiva de espécies na estação subseqüente. Na primavera de 2005 , ano em que o defeso do arrasto ocorreu entre abril e junho, os indicadores reprodutivos também foram expressivos, comparáveis àqueles do verão de 2007. As espécies com valores mais elevados de IAR não foram as mesmas nas duas estações: ao mudar-se o período de defeso, mudaram também as espécies de peixes com melhores indicadores reprodutivos. Conclui-se que fora do defeso a pesca de arrasto sempre incidirá sobre uma parcela da ictiofauna em reprodução, em grau menor no inverno.

Face ao exposto, recomenda-se que se mantenha o defeso na primavera, o que beneficiará não apenas as espécies de peixes que desovam na própria primavera, mas também aquelas que se preparam para desova no verão. Simultaneamente alerta-se para a necessidade do controle do esforço da pesca de arrasto camaroeiro no verão, de maneira que não seja capturada uma elevada quantidade de peixes desovantes que comprometa a manutenção dos estoques.

\section{AGRADECIMENTOS}

Aos membros da comunidade pesqueira de Enseada, São Francisco do Sul, Santa Catarina, e aos três consultores que, avaliando o trabalho para a Revista, contribuíram para a versão final. Projeto realizado com suporte financeiro do CNPq.

\section{REFERÊNCIAS BIBLIOGRÁFICAS}

Agostinho, A.A.; V.P. Mendes; H.I. Suzuki \& C. Canzi. 1993. Avaliação da atividade reprodutiva da comunidade de peixes dos primeiros quilômetros a jusante do reservatório de Itaipu. Unimar 15 (Supl.): 175-189.

Berghahn, R.; M. Waltemath \& A.D. Rundsorp. 1992. Mortality of fish from the by-catch of shrimp vessels in the North Sea. Journal of Applied Ichthyology 8: 293-306.

Blaber, S.J.M. 2000. Tropical Estuarine Fishes. Oxford, Blackwell Science, 372p.

Branco, J.O. \& J.R. Verani. 2006. Análise quali-quantitativa da ictiofauna acompanhante na pesca do camarão sete-barbas na Armação do Itapocoroy, Penha, Santa Catarina. Revista Brasileira de Zoologia 23 (2): 381-391.

Cervigón, F.; R. Cipriani; E. Fischer; L. Garibaldi; M. Hendrickx; A.J. Lemus; R. Márquez; J.M. Poutiers; G. Robaina \& B. Rodriguez. 1992. Especies comerciales marinas y de aguas salobres de la costa septentrional de Sur America. Roma, FAO, 513p. 
Chaves, P.T. \& A.L. Vendel. 1997a. Indicadores reprodutivos das espécies de Citharichthys Bleeker (Teleostei, Pleuronectiformes) na Baía de Guaratuba, Paraná, Brasil. Revista Brasileira de Zoologia 14 (1): 73-79.

Chaves, P.T. \& A.L. Vendel. 1997b. Reprodução de Stellifer rastrifer (Jordan) (Teleostei, Sciaenidae) na Baía de Guaratuba, Paraná, Brasil. Revista Brasileira de Zoologia 14 (1): 81-89.

Chaves, P.T. \& C.E. CorrêA. 2000. Temporary use of a coastal ecosystem by fish: Pomadasys corvinaeformis (Perciformes: Haemulidae) at Guaratuba Bay, Brazil. Revista Brasileira de Oceanografia 48 (1): 1-7.

Chaves, P.T. \& J.-L. Bouchereau. 2000. Use of mangrove habitat for reproductive activity by the fish assemblage in the Guaratuba Bay, Brazil. Oceanologica Acta 23 (3): 273-280.

Chaves, P.T. \& M.C. Robert. 2003. Embarcações, artes e procedimentos da pesca artesanal no litoral sul do Estado do Paraná, Brasil. Atlântica 25 (1): 53-59.

Chaves, P.T.; A. Rickli \& J.-L. Bouchereau. 1998. Strategie d'occupation de la mangrove de la baie de Guaratuba (Brésil) par le prédateur Isopisthus parvipinnis (Teleostei). Cahiers de Biologie Marine 39 (1): 63-71.

Chaves, P.T.; G. Cova-Grando \& C. Calluf. 2003. Demersal ichthyofauna in a continental shelf region on the south coast of Brazil exposed to shrimp trawl fisheries. Acta Biologica Paranaense 32 (1-4): 69-82.

CorrêA, C.E.; P.T.C. Chaves \& P.R. Bittencourt. 2005. Biology of Chirocentrodon bleekerianus (Poey, 1867) (Clupeiformes: Pristigasteridae) in a Continental Shelf Region of Southern Brazil. Brazilian Archives of Biology and Technology 48 (3): 419-427.

Diamond, S.L.; L.B. Crowder \& L.G. Cowell. 1999. Catch and bycatch: the qualitative effects of fisheries on population vital rates of Atlantic croaker. Transactions of the American Fisheries Society 128: 1085-1105.

Diamond, S.L.; L.G. Cowell \& L.B. Crowder. 2000. Population effects of shrimp trawl bycatch on Atlantic croaker. Canadian Journal of Fisheries Aquatic Science 57: 20102021.

Figueiredo, J.L. \& N.A. Menezes, 1978. Manual de Peixes Marinhos do Sudeste do Brasil. II. Teleostei (1). São Paulo, Museu de Zoologia da Universidade de São Paulo, 110p.

Figueiredo, J.L. \& N.A. Menezes, 1980. Manual de Peixes Marinhos do Sudeste do Brasil. III. Teleostei (2). São Paulo, Museu de Zoologia da Universidade de São Paulo, 90p.

Figueiredo, J.L. \& N.A. Menezes, 2000. Manual de Peixes Marinhos do Sudeste do Brasil. VI. Teleostei (5). São Paulo, Museu de Zoologia da Universidade de São Paulo, 116p.
Fishbase. Disponível em: http://www.fishbase.org/search.php [Acessado em 26.IV.2007].

Gomes, I.D. \& P.T. Chaves. 2006. Ictiofauna integrante da pesca de arrasto camaroeiro no litoral sul do Estado do Paraná. Bioikos 20 (1): 9-13.

Hill, B.J. \& T.J. WASSENBERg. 2000. The probable fate of discards from prawn trawlers fishing near coral reefs - a study in the northern Great Barrier Reef, Austrália. Fisheries Research 48: 277-286.

KotAS, J.E. 1998. Fauna Acompanhante nas Pescarias de Camarão em Santa Catarina. Brasília, Ibama, Coleção MeioAmbiente, Série Estudos Pesca 24, 76p.

Menezes, N.A. \& J.L. De Figueiredo. 1980. Manual de Peixes Marinhos do Sudeste do Brasil. IV. Teleostei (3). São Paulo, Museu de Zoologia da Universidade de São Paulo, 96p.

Menezes, N.A.; P.A. Buckup; J.L. de Figueiredo \& R.L. de Moura. 2003. Catálogo das espécies de peixes marinhos do Brasil. São Paulo, Museu de Zoologia da Universidade de São Paulo, 160p.

PAIVA, M.P. 1997. Recursos pesqueiros estuarinos e marinhos do Brasil. Fortaleza, Universidade Federal do Ceará Editora, $278 \mathrm{p}$.

Paiva-Filho, A.M. \& J.M. Schmiegelow. 1986. Estudo sobre a ictiofauna acompanhante da pesca do camarão sete-barbas (Xiphopenaeus kroyeri) nas proximidades da Baía de Santos, SP. I - Aspectos quantitativos. Boletim do Instituto de Pesca 34: 79-85.

Perez, J.A.A. \& P.R. Pezzuto. 1998. Valuable shellfish species in the by-catch of shrimp fishery in southern Brazil: spatial and temporal patterns. Journal of Shellfish Research 17: 303-309.

Robert, M.C. \& P.T. Chaves. 2001. Observações sobre o ciclo de vida da corvina, Micropogonias furnieri (Desmarest) (Teleostei, Sciaenidae), no litoral do Estado do Paraná, Brasil. Revista Brasileira de Zoologia 18 (2): 421-428.

VAZzoler, A.E.A. de M. 1996. Biologia da reprodução de peixes teleósteos. Teoria e prática. Maringá, EDUEM/SBI, 196p.

Vendel, A.L. \& P.T. Chaves. 2006. Use of an estuarine environment (Barra do Saí lagoon, Brazil) as nursery by fish. Revista Brasileira de Zoologia 23 (4): 1117-1122.

Vieira, J.P.; M.C. Vasconcellos; R.E. Silva \& L.G. Fisher. 1996. A ictiofauna acompanhante da pesca do camarão-rosa (Penaeus paulensis) no estuário da Lagoa dos Patos, RS. Atlântica 18: 123-142.

WALTER, U. \& P.H. BeCKer. 1994. The significance of discards from brown shrimp fisheries for seabirds in the Wadden Sea preliminary results. Ophelia (Suppl.): 253-262.

Recebido em 14.VIII.2007; aceito em 26.XI.2007. 